\title{
O Impacto dos Inibidores da HMG-CoA Redutase na Modificação da História Natural da Doença Coronária
}

\author{
Sergio Emanuel Kaiser, Monica Luiza de Alcantara \\ Rio de Janeiro, RJ
}

No ultimo congresso da American Heart Association, em 1997, foram divulgados os resultados de dois estudos prospectivos multicêntricos de prevenção primária e secundária de eventos cardiovasculares através do uso a longo prazo de drogas inibidoras da HMG-CoA redutase, respectivamente, o estudo AF/Texcaps, comparando lovastatina e placebo em pacientes aparentemente saudáveis e o estudo LIPID, avaliando a eficácia da pravastatina frente ao placebo em pacientes com episódios anteriores de infarto do miocárdio (IM) ou angina instável.

As conclusões preliminares geradas por esses dois importantes estudos, somadas aos resultados amplamente conhecidos dos três ensaios clínicos precedentes ${ }^{1-3}$, parecem completar as franjas de um amplo espectro de indicações para o emprego de um grupo de medicamentos que não somente mudou a história natural da doença coronária, como permitiu confirmar através de prova terapêutica, a importância dos conceitos recém adquiridos sobre a composição da placa aterosclerótica e seu papel na precipitação de acidentes coronários agudos. A partir da publicação desses novos resultados em periódicos, a comunidade médica disporá de numeroso banco de informações, abrangendo quase 150 mil pacientes/ano de acompanhamento prospectivo, constituindo-se em fonte para metanálises estatisticamente robustas em diversos subgrupos de risco.

Com base nas evidencias atualmente disponíveis, este artigo tem por objetivo: a) sumarizar os resultados dos cinco estudos de prevenção primária e secundária com inibidores da HMG-CoA redutase já completados; b) tecer considerações sobre os mecanismos de ação já conhecidos, aqueles ainda a explorar e as similaridades e diferenças entre as vastatinas existentes; c) redefinir, com base nestes resultados, as indicações e os subgrupos mais beneficiados pelo emprego das vastatinas na prevenção primária e secundária da doença coronária.

Todos os cinco grandes estudos foram prospectivos, placebo-controlados, aleatorizados, do tipo "intenção de tratar". A tabela I e a figura 1 sumarizam as principais características.

Universidade do Estado do Rio de Janeiro - Clinex

Correspondência: Sergio Emanuel Kaiser - Rua Visconde Silva, 52 - S/705 - 22271090 - Rio de Janeiro, RJ

Recebido para publicação em 20/2/98

Aceito em 13/5/98

\section{Estudos de prevenção primária com inibidores da HMG-CoA redutase}

West of Scotland Coronary Prevention Study (WOSCOPS) -O fármaco empregado foi a pravastatina na dose de 40mg diários e o período de acompanhamento estendeu-se por quase cinco anos, findos os quais, observou-se uma redução altamente significativa no risco de ocorrência do desfecho primário, comparativamente ao grupo placebo (fig. 1). Reduções significativas foram observadas no risco relativo de infarto não fatal $(\mathrm{p}<0,001)$ e na mortalidade global. Outros desfechos secundários, como mortalidade cardiovascular em geral e procedimentos de revascularização, também foram expressivamente reduzidos. Os benefícios independeram de faixa etária, tabagismo ou níveis prévios de HDLc ou triglicerídeos. Não foram observadas quaisquer diferenças relativamente ao grupo placebo, na ocorrência de mortes violentas ou por câncer.

The Air Force/Texas Coronary Atherosclerosis Prevention Study (AF/TEXCAPS) - Os dados preliminares deste estudo foram apresentados na última reunião da American Heart Association e serão ainda objeto de publicação e análises mais detalhadas.

O objetivo primário desse projeto procurou responder a dúvida sobre a eficácia a longo prazo de um inibidor da HMG-CoA redutase, no caso a lovastatina, em reduzir a taxa de um $1^{\circ}$ ataque cardíaco em indivíduos assintomáticos, com perfil lipídico mais representativo daquele encontrado na população geral.

Os níveis médios de lipídeos eram representados por um colesterol total de $225 \mathrm{mg} / \mathrm{dL}$, LDLc de $145 \mathrm{mg} / \mathrm{dL}$ (130$190 \mathrm{mg} / \mathrm{dL}$ ) e HDLc $<50 \mathrm{mg} / \mathrm{dL}$. A grande maioria dos pacientes exibia dois ou menos fatores de risco coronário. A lovastatina foi administrada, àqueles recrutados para seu emprego, na dose de 20 a 40mg, com valores médios em torno de $30 \mathrm{mg}$. A redução do colesterol total foi de $18 \%$, a do LDLc de $25 \%$ e a dos triglicerídeos de $15 \%$, mantida ao longo do estudo. Após 4,8 anos, o comitê independente de análise dos resultados recomendou sua interrupção face às evidencias de benefício geradas pelo uso do fármaco (tab. I e fig. 1). A redução no risco de um desfecho primário em relação ao grupo placebo foi também expressiva nos subgrupos de fumantes $(59 \%)$ hipertensos $(43 \%)$ diabéticos (43\%) mulheres (54\%) homens (34\%) e idosos > 65 anos 
(29\%). Com bases nesses resultados, os autores concluíram a apresentação deste estudo recomendando "o uso de 20 a $40 \mathrm{mg}$ de lovastatina, associadas a dieta e exercícios físicos em homens $>45$ anos e mulheres $>55$ anos com níveis de LDLc $>130 \mathrm{mg} / \mathrm{dLeHDLc}<50 \mathrm{mg} / \mathrm{dL}$ '.

Estudos de prevenção secundária com inibidores da HMG-CoA redutase

Scandinavian Simvastatin Survival Study (4S) - Um dos mais conhecidos, primeiro no gênero a ser publicado, em 1994, este estudo recrutou prospectivamente, pacientes entre 35 a 70 anos com níveis elevados de colesterol total, sobreviventes de IM ou angina instável (tab. I). O desfecho primário pré-especificado foi a mortalidade total. "Eventos coronários maiores", definidos como morte coronária, infarto agudo miocárdio (IAM) não fatal certo ou provável, e parada cardíaca ressuscitada, responderam pelo desfecho secundário. Inicialmente administrada na dose de $20 \mathrm{mg}$, a sinvastatina foi posteriormente fornecida a $37 \%$ dos pacientes na dose de $40 \mathrm{mg} /$ dia. A redução média dos níveis de colesterol, LDLc e triglicerídeos, mantida ao longo do estudo, foi de, respectivamente, $25 \%, 35 \%$ e $10 \%$, ao passo que o HDLc elevou-se cerca de $8 \%$.

Após 5,4 anos de acompanhamento, observavam-se benefícios significativos para ambos os sexos e naqueles $>$ e $<60$ anos. Análise posterior revelou benefício semelhante

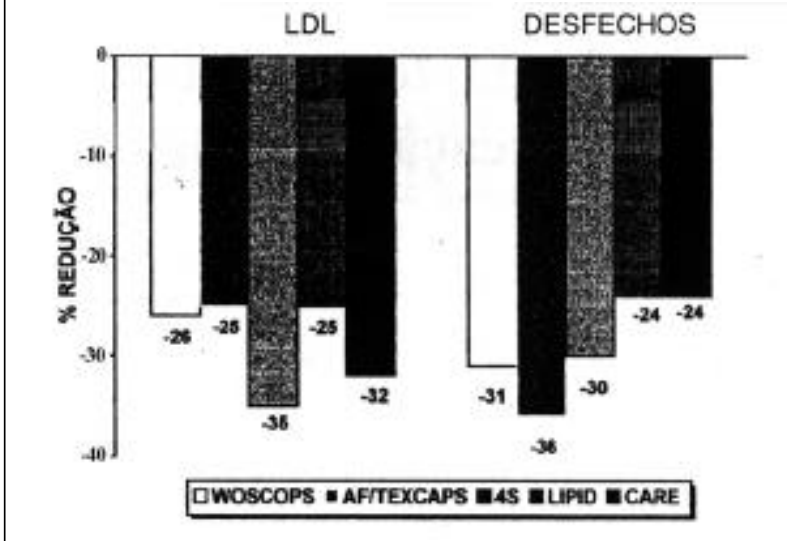

Fig. 1 - Gráfico demonstrativo, nos cinco grandes estudos de prevenção primária e secundária, das reduções percentuais nos níveis de LDLc obtidas com vastatinas e nos desfechos primários pré-especificados.

gerado pelo uso de sinvastatina independentemente dos níveis basais de HDLc e triglicerídeos, assim como ao longo de quatro diferentes categorias de indivíduos estratificados segundo níveis crescentes de LDLc ${ }^{4}$.

Cholesterol and Recurrent Events (CARE) Study-Os integrantes deste estudo eram homens e mulheres com idade média de 59 anos, sobreviventes de um IM três a 20 meses antes do ingresso no estudo. O nível plasmático de colesterol total era $<240 \mathrm{mg} / \mathrm{dL}$, os triglicerídeos $<350 \mathrm{mg} / \mathrm{dL}$

\begin{tabular}{|c|c|c|c|c|c|c|c|}
\hline ESTUDO & $\begin{array}{l}\text { Níveis de } \\
\text { LDL } \\
\text { colesterol } \\
(\mathrm{mg} / \mathrm{dl})\end{array}$ & $\begin{array}{l}\text { Núme ro } \\
\text { recrutado }\end{array}$ & $\begin{array}{c}\text { Proporção de } \\
\text { mulheres }\end{array}$ & $\begin{array}{c}\text { Desfecho } \\
\text { primário }\end{array}$ & $\begin{array}{l}\text { Tempode } \\
\text { aparecimento } \\
\text { do efeito } \\
\text { (meses) }\end{array}$ & $\begin{array}{c}\text { Medicação } \\
\text { cardíaca } \\
\text { concomitante }\end{array}$ & $\begin{array}{l}\text { NNT*e } \\
\text { IC } 95 \%\end{array}$ \\
\hline \multicolumn{8}{|l|}{$\begin{array}{l}\text { Prevenção } \\
\text { primária }\end{array}$} \\
\hline WOSCOPS & $155-232$ & 6595 & zero & $\begin{array}{l}\text { IAM não fatal } \\
\text { + morte } \\
\text { coronária }\end{array}$ & 6 & $\begin{array}{c}\text { Nenhuma } \\
\text { expressivamente }\end{array}$ & $41(29-74)$ \\
\hline AF/TEXCAPS & $130-190$ & 6605 & $997(15 \%)$ & $\begin{array}{c}\text { Angina } \\
\text { instável + } \\
\text { IAMfatal+ } \\
\text { IAM não fatal } \\
\text { + morte súbita }\end{array}$ & 13 & $\begin{array}{c}\text { Nenhuma } \\
\text { expressivamente }\end{array}$ & $24 \#$ \\
\hline \multicolumn{8}{|l|}{$\begin{array}{l}\text { Prevenção } \\
\text { secundária }\end{array}$} \\
\hline $4 \mathrm{~S}$ & $\begin{array}{c}215-310 \\
\text { (Colesterol } \\
\text { total) }\end{array}$ & 4444 & $827(18.6 \%)$ & $\begin{array}{c}\text { Mortalidade } \\
\text { total }\end{array}$ & 18 & $\begin{array}{c}\text { AAS em 37\% } \\
\text { Betaem } 57 \% \\
\text { Nitratos em 33\% }\end{array}$ & $27(20-55)$ \\
\hline CARE & $115-174$ & 4159 & $583(14 \%)$ & $\begin{array}{l}\text { Mortalidade } \\
\text { coronária + } \\
\text { IAM não fatal }\end{array}$ & 24 & $\begin{array}{c}\text { AAS em } 83 \% \\
\text { Betaem } 40 \% \\
\text { Nitratos em } 32 \%\end{array}$ & $32(12-46)$ \\
\hline LIPID & $\begin{array}{c}155-251 \\
\text { (colesterol } \\
\text { total) }\end{array}$ & 9014 & $1516(17 \%)$ & $\begin{array}{c}\text { Mortalidade } \\
\text { coronária }\end{array}$ & 15 & $\begin{array}{c}\text { AAS em } 82 \% \\
\text { Beta em } 47 \% \\
\text { IECAem } 16 \% \\
\text { Nitratos em } 36 \%\end{array}$ & $26(20-31)$ \\
\hline
\end{tabular}


e os níveis de LDLc situaram-se entre 115 e $174 \mathrm{mg} / \mathrm{dL}$. As concentrações médias basais de colesterol total e LDLc eram de, respectivamente, 209 e $139 \mathrm{mg} / \mathrm{dL}$.

$\mathrm{Na}$ dose de $40 \mathrm{mg}$ diários, a pravastatina modificou favoravelmente o risco de um desfecho primário em $24 \%$ ao fim de cinco anos de acompanhamento (tab. I e fig. 1). Ao longo do estudo, a pravastatina reduziu os níveis de colesterol total, LDLc e triglicerídeos em, respectivamente, $20 \%, 28 \%$ e $14 \%$, tendo elevado o HDLc em cerca de $5 \%$.

Os desfechos secundários experimentaram uma redução significativa de risco: o de infarto não fatal foi reduzido em 23\%, a necessidade de revascularização miocárdica em $27 \%$ e o risco de acidente vascular cerebral (AVC) em $31 \%$, dado particularmente importante, por se tratar do $1^{\circ}$ estudo do gênero em que este desfecho foi pré-especificado. Não se observaram diferenças significativas na mortalidade global ou não cardiovascular. Os resultados favoráveis à pravastatina foram consistentes ao longo de vários subgrupos, como fumantes, hipertensos, diabéticos, pacientes previamente revascularizados, indivíduos com disfunção ventricular esquerda, idosos entre 60 e 75 anos, homens e mulheres. Nestas, a redução de risco foi da ordem de $46 \%$, contrastando com um valor de $20 \%$ para os homens.

Long-term Intervention with Pravastatin in Ischemic Disease (LIPID) Study - Os resultados preliminares deste estudo prospectivo, aleatório, placebo-controlado, foram apresentados na última reunião da American Heart Association. Desenhado de forma semelhante ao estudo CARE, diferiu deste essencialmente em função do maior número de pacientes recrutados e pela inclusão de pessoas com episódios prévios de angina instável, sem infarto prévio. A população alvo, deveria ter níveis de colesterol total dentro da média encontrada nas sociedades industrializadas, entre 155 e $251 \mathrm{mg} \%$ e níveis de triglicerídeos $<455 \mathrm{mg} / \mathrm{dL}$.

Os sobreviventes de IAM compunham $2 / 3$ do total. $\mathrm{O}$ desfecho primário pré-especificado foi a mortalidade por doença isquêmica e os desfechos secundários principais foram a incidência de doença isquêmica fatal e infarto não fatal, revascularização miocárdica e AVC (fig. 1, tab. I).

No decorrer do estudo, a pravastatina na dose de $40 \mathrm{mg}$ diários reduziu os níveis de colesterol total, LDL e triglicerídeos em, respectivamente, $18 \%, 25 \%$ e $12 \%$ ao passo que o HDLc elevou-se em $6 \%$.

O comitê de segurança recomendou, em 1997, a suspensão do estudo face aos benefícios evidentes conferidos pelo emprego do medicamento. Relativamente ao grupo placebo, o risco de um desfecho primário foi significativamente reduzido em $24 \%$. As mortes coronárias mais infarto não fatal e o AVC foram reduzidos pela pravastatina em, respectivamente, $24 \%$ e $20 \%$. Reduções estatisticamente significantes de $18 \%, 16 \%$ e $24 \%$ foram observadas para a necessidade de revascularização cirúrgica, angioplastia e mortalidade total. A incidência de câncer, por sua vez, foi semelhante nos dois grupos.

\section{Possíveis mecanismos responsáveis pela ação benéfi- ca dos inibidores da HMG-CoA redutase}

Em 1993, o National Cholesterol Education Program, ao rever as recomendações referentes aos níveis ideais de LDL-colesterol (LDLc) a serem atingidos através de terapia orientada para a prevenção secundária da doença coronária, reduziu de 130 para $100 \mathrm{mg} / \mathrm{dL}$ o limite considerado aceitável para impedir a progressão da aterosclerose ${ }^{5}$. Este novo limiar, endossado pelo $2^{\circ}$ Consenso Brasileiro sobre Dislipidemias ${ }^{6}$ foi estabelecido com base numa série de estudos com desfechos angiográficos especificados, onde se constatou a necessidade de reduzir agressivamente os níveis plasmáticos desta lipoproteína, a fim de não só deter a evolução do processo, como também de promover algum grau de regressão das lesões já formadas ${ }^{7}$. Uma série de evidências indiretamente obtidas por de cintigrafiamiocárdica ${ }^{8,9}$, ou métodos de investigação da função endotelial ${ }^{10,11}$ porém, já evidenciava a melhora de vários indicadores clínicos e funcionais, muito antes de se detectar qualquer sinal de regressão nas lesões ateroscleróticas. Documentava-se também a pronta restauração da função vasodilatadora do endotélio vascular tanto na circulação coronária ${ }^{12,13}$ como na periféri$\mathrm{ca}^{14}$, através do uso de vastatinas isoladamente ou em associação com terapia antioxidante ${ }^{15}$. Não surpreende, portanto, a atribuição da rápida melhora clínica dos pacientes coronários a uma melhora global da capacidade de vasodilatação arterial e arteriolar. Estas evidências, no entanto, pareciam quando muito, reforçar o vínculo entre a ação favorável do medicamento e sua capacidade de reduzir os lipídeos plasmáticos, antecipando alguns benefícios agregados ao mais desejado e ambicioso - a redução da morbimortalidade coronária - cuja possibilidade já se fazia sentir através de análises post-hoc dos estudos de desfecho angiográfico ${ }^{7}$. Recentemente, no entanto, O' Driscoll e col notaram a ausência de correlação entre a melhora da função endotelial, obtida em apenas quatro semanas após o início de terapia com sinvastatina e a queda dos níveis séricos de LDLc, acenando com a possibilidade de efeitos desvinculados da redução do $\mathrm{LDLc}^{16}$.

Uma das mais intrigantes sugestões de possíveis benefícios não ligados à redução do colesterol proporcionado pela terapia com vastatinas, foi aventada somente após a publicação dos primeiros estudos prospectivos, que documentavam a redução de eventos cardiovasculares, num período de seis a 18 meses, após o início do tratamento: o estudo $\mathrm{POSCH}^{17}$ valendo-se de cirurgia de bypass ileal como forma de obter controle dos níveis de LDLc, oferecia uma rara oportunidade de documentar efeitos de intensidade similar à obtida com vastatinas, com a vantagem de independer de aderência a qualquer medicação. No entanto, foram necessários nove anos de acompanhamento até que algum benefício na redução de morbimortalidade fosse observado!

Uma análise subseqüente do estudo WOSCOPS trouxe à luz mais alguns interessantes dados, sugerindo fortemente a existência de benefícios adicionais conferidos pelo 
emprego de vastatinas, além dos resultantes unicamente do restabelecimento de níveis "normais" de LDLc. Os participantes deste estudo recrutados para o grupo "pravastatina" foram subdivididos em cinco categorias ou percentis, de acordo com a intensidade da redução do LDLc obtida através do uso do fármaco. A análise da relação entre os níveis de lipoproteína atingidos com o tratamento e a morbimortalidade cardiovascular, não revelou nenhum benefício adicional conferido pela redução do LDLc a mais de $20 \%$ dos valores basais ${ }^{18,19}$. Nesse mesmo estudo, os autores compararam o percentual de eventos nos grupos "placebo" e "pravastatina" com os níveis equivalentes de colesterol derivados do modelo de previsão de risco do estudo de Framingham ${ }^{20}$. No grupo placebo, a ocorrência de eventos correlacionou-se aos diferentes percentis de LDLc de forma superponível à curva derivada do modelo de Framingham, ao passo que no grupo pravastatina, os mesmos percentis de LDLc correlacionavam-se a uma ocorrência de eventos sistematicamente menor. Em outras palavras, enquanto a redução de risco relativo prevista pela alteração dos níveis plasmáticos no modelo era de $22 \%$, a redução de risco obtida nos pacientes do grupo pravastatina era de $46 \%$. Ainda nesta comunicação foi apresentado o resultado de uma análise de superposição que consistia em examinar a incidência de eventos durante o período do estudo, numa faixa de pacientes com nível semelhante de LDLc, entre 150 e 170mg/dL, já em uso do fármaco ou placebo correspondente. Foram desta forma identificados 466 pacientes do grupo pravastatina com LDLc médio de $159 \mathrm{mg} / \mathrm{dL}$ e 446 do grupo placebo com LDLc médio de $159 \mathrm{mg} / \mathrm{dL}$ ( $\mathrm{p}=\mathrm{NS}$ ). Ao fim do estudo, após correção para fatores de risco basais, os pacientes recrutados para o uso de pravastatina neste subgrupo exibiam uma taxa de eventos $47 \%$ inferior $(\mathrm{p}<0,041)$ àquela observada para os indivíduos do outro grupo, apesar da equivalência entre os níveis de LDLc atingido.

Efeito sobre o metabolismo das IDL e outras funções celulares - Ao bloquear no hepatócito a conversão da HMG-CoA em mevalonato, as vastatinas limitam numerosas etapas subsequentes na cadeia de biossíntese do colesterol, incluindo a síntese das IDL, remanescentes altamente aterogênicos das VLDL, ricas em apo B-100 ${ }^{21,22}$. Tal atributo é exclusivo desta classe de redutores do colesterol.

A atividade das vastatinas limita a velocidade de síntese não somente do colesterol, mas também de uma gama de moléculas envolvidas em funções como respiração celular e transdução de sinais. Portanto, estes fármacos poderiam modificar outros constituintes do meio vascular, com repercussões ainda não totalmente esclarecidas sobre o processo aterosclerótico ${ }^{23}$.

Ação estabilizadora da placa -Uma série de processos de natureza hemostática e inflamatória, profundamente ligados à instabilização da placa aterosclerótica, parecem ser afetados pelos inibidores da HMG-CoA redutase. Estudo em modelos ex-vivo empregando a pravastatina por 2 a 3 meses indicaram redução da formação de trombo plaquetário, possivelmente secundária à limitação da síntese de tromboxane $\mathrm{A}_{2}$ induzida pela droga ${ }^{24}$. Algumas vastatinas parecem inibir a interação entre plaquetas e fibrinogênio sérico ${ }^{25}$ e reduzir a expressão de vários agentes envolvidos na cascata de coagulação ${ }^{26}$.

Crescentes evidências apóiam a existência de efeito antiinflamatório possivelmente mediado por a) ação antiproliferativa ${ }^{27}$, b) bloqueio da ativação de macrófagos e formação de células espumosas ${ }^{28,29}$ e c) inibição da atividade do fator de crescimento derivado de plaquetas ${ }^{30}$, um poderoso estimulante da proliferação de células musculares lisas ${ }^{31}$. Em doses habituais in vitro, as vastatinas também modulariam a função imune, alterando a regulação da transcrição do DNA, a atividade citotóxica de células killer e inibindo a citotoxicidade celular dependente de anticorpos ${ }^{32}$.

Também tem sido documentada a modificação do potencial oxidativo das LDL induzida por vastatinas, sinalizando, pois, a participação de efeito antioxidante ${ }^{33,34,35}$.

As diferenças entre os inibidores da HMG-CoA redutase revestem-se de relevância clínica? -Existem atualmente seis compostos em uso clínico, todos disponíveis no Brasil ou com lançamento previsto para breve.

A literatura está repleta de estudos comparativos diretos e indiretos entre os principais compostos, sumarizados em parte por pelo menos uma metanálise de porte, reunindo as diversas informações recolhidas de 52 estudos duplo$\operatorname{cegos}^{36}$. Relativamente à potência, observa-se em todos os compostos uma relação dose-efeito ${ }^{36,37}$, tornando possível, ainda que a custos diferentes, reduções comparáveis do LDLc mediante ajuste da equivalência de doses quase sem comprometimento da tolerabilidade. A atorvastatina exibe as reduções mais expressivas do LDLc a doses equivalentes, baixando também significativamente os triglicerídeos, motivo pelo qual representa a escolha ideal para tratamento de hipercolesterolemia familiar ${ }^{38}$. Pelo menos dois estudos independentes ${ }^{39,40}$ evidenciaram uma interessante propriedade da fluvastatina, cuja potência aumentaria em cerca de $13 \%$ para cada incremento de $100 \mathrm{mg} / \mathrm{dL}$ na concentração basal de LDLc, propriedade capaz de favorecer também a escolha deste fármaco em pacientes com hipercolesterolemia mais grave.

Tentativas de comparação entre os diversos agentes exibem considerável heterogeneidade relativamente às ações das vastatinas sobre influências trombóticas e hemostáticas, cuja relevância clínica parece sedimentada. Metodologias diversas, diferentes critérios de aferições, diferentes perfis clínicos de pacientes, número de casos insuficientes e trabalhos institucionais sem confirmação multicêntrica criam um terreno bastante movediço, especialmente vulnerável a pressões da indústria farmacêutica, preocupada, admissivelmente, em valorizar seus respectivos produtos na busca pela maior fatia possível de um gigantesco mercado consumidor.

Considerações mercadológicas à parte, é fato que até o presente momento, somente três destes fármacos foram testados em estudos prospectivos, aleatorizados e placebocontrolados, dentre os quais responde a pravastatina por cerca de dois terços dos 150 mil pacientes/ano de observa- 
ção. Muito embora as seis vastatinas disponíveis pareçam compartilhar propriedades comuns, seria preferível reservar o uso dos fármacos ainda não amplamente testados a situações mais específicas, como por exemplo, prescrever a fluvastatina àqueles menos providos de recursos em função do mais baixo custo ou recomendar o uso de atorvastatina a pacientes com hipertrigliceridemia associada.

Deve-se buscar a redução absoluta ou percentual do LDL-colesterol? - Há cerca de oito anos, anteriormente à era das vastatinas, Holme ${ }^{41}$ concluía através de metanálise abrangendo os 15 estudos de intervenção existentes, que, para cada redução percentual do colesterol, a correspondente redução de eventos era menor em populações com níveis basais de colesterol mais baixos, aumentando exponencialmente com o aumento de suas concentrações plasmáticas. Conseqüentemente, o benefício clínico advindo da redução do LDLc seria desigual, concentrandose na margem superior da curva de distribuição dos níveis lipídicos basais, em concordância com a morfologia curvilínea entre concentração de colesterol e risco cardiovascular definida pelo estudo MRFIT ${ }^{42}$.

Semelhante conclusão pareceu emergir de uma análise dos estudos com desfecho angiográfico, onde o benefício resultante de grandes reduções do LDLc era bem mais nítido em pacientes com elevados níveis séricos basais de lipídeos, ao contrário daqueles com colesterol apenas moderadamente ou pouco elevado, onde não se fazia notar regressão substancial da aterosclerose com o tratamento ${ }^{43}$.

O estudo CARE, por sua vez, analisando desfechos clínicos, não evidenciou benefícios significativos naqueles indivíduos com concentrações basais de LDLc $<125 \mathrm{mg} / \mathrm{dL}^{44}$. Neste caso, diferenças significativas em favor da vastatina talvez até existam, mas para documentá-las, teria sido necessário tratar um maior número de pacientes, uma vez que o benefício absoluto em indivíduos com níveis mais baixos de LDLc seria necessariamente menor.

Com relação ao objetivo ideal a ser atingido com a terapêutica, recente metanálise abrangendo 34 estudos de prevenção secundária não encontrou evidência de um nível limiar para redução do LDLc - em outras palavras - em coronariopatas, quanto menor o LDLc, melhor ${ }^{45}$. Em apoio a esta tese, um estudo envolvendo pacientes revascularizados com pontes de safena só demonstrou interrupção do processo aterosclerótico nos enxertos, naqueles cujos níveis de LDLc foram agressivamente reduzidos a menos de $100 \mathrm{mg} / \mathrm{dL}^{46}$.

A fim de determinar a correlação entre a magnitude dos níveis atingidos durante o tratamento e a intensidade do benefício obtido, os autores do estudo $4 \mathrm{~S}$ dividiram os integrantes do ensaio em três categorias de redução percentual de LDLc produzida por sinvastatina: abaixo de $34 \%$, entre 34 e $44 \%$ e entre 44 e $70 \%$. As respectivas reduções no risco de um desfecho primário foram $10 \%, 13,3 \%$ e $18,9 \%$. Por outro lado, como já comentado anteriormente, os investigadores do WOSCOPS e, segundo publicação recentemente divulgada, também os responsáveis pelo estudo CARE não observaram benefícios comparavelmente maiores mediante reduções percentuais do LDLc superiores a $20 \%$ dos valores basais ${ }^{19,48}$.

Vale ressaltar, este gênero de análise de subgrupos oriundos de estudos prospectivos funciona como boa fonte geradora de hipóteses, especialmente quando biologicamente plausíveis, mas carece de poder para determinar mudanças de conduta. Por este motivo, deve prevalecer entre nós a recomendação do Consenso Brasileiro ${ }^{6}$, ao menos até o surgimento de novos estudos prospectivos desenhados para responder especificamente a esta questão. Ou seja, em prevenção primária, buscar um LDLc $<130 \mathrm{mg} / \mathrm{dL}$ e em prevenção secundária $<100 \mathrm{mg} / \mathrm{dL}$.

O elevado custo para nós, brasileiros, das doses de pravastatina empregadas nos grandes estudos nos impele necessariamente a perguntar se doses menores não exerceriam efeito similar. Infelizmente não há resposta concreta a esta indagação, especialmente se for levada em conta a participação dos efeitos não ligados à redução dos lipídeos. Traduzindo favoravelmente a eficácia de doses menores, mencione-se recente estudo prospectivo aleatorizado de 665 pacientes com idade média de 73 anos, onde um grupo foi tratado com 10 a $20 \mathrm{mg}$ de pravastatina e outro com doses ainda menores, de $5 \mathrm{mg}$. A redução média de eventos cardiovasculares foi de $33,5 \%$ para um decréscimo de $20 \mathrm{mg} /$ dL no LDLc e chegou a $27 \%$ naqueles $>$ de $75 \operatorname{anos}^{49}$.

Terapia hipolipemiante embasada em evidência - Os estudos prospectivos aleatorizados placebo-controlados representam a forma mais confiável para validação de uma proposta terapêutica, mas a aplicação de seus resultados no dia-a-dia requer certos cuidados, pois a estimativa do benefício absoluto e o custo-eficácia da terapia estudada podem tornar sua aplicabilidade bastante restrita. Ensaios clínicos deste porte requerem critérios de seleção muito restritivos, excluindo freqüentemente os pacientes mais idosos, mais enfermos ou aqueles portadores de comorbidades, donde resulta por fim, uma amostra nem sempre representativa da população encontrada no dia-a-dia do médico.

Como seria esperado, as vastatinas exercem sua ação preventiva máxima nos grupos de maior risco de eventos, como os hipercolesterolêmicos sobreviventes de um episódio coronário ${ }^{50}$, cujo risco de eventos é três a seis vezes superior ao de uma população saudável com níveis semelhantes de lipídeos séricos ${ }^{51,52}$. Portanto, como demonstra o exemplo da figura 2, o NNT $*$, ou seja, o número de pacientes necessários para tratar com sinvastatina por um período equivalente à duração do estudo $4 \mathrm{~S}$ para impedir a ocorrência de um desfecho primário, foi equivalente a quase a $6^{\mathrm{a}}$ parte do NNT para a pravastatina num subgrupo de pacientes "saudáveis" do WOSCOPS, entre 45-54 anos, cujo único fator de risco era a hipercolesterolemia.

Há de se tomar, também, cautela ao tentar estabelecer comparações entre a eficácia relativa dos diversos fármacos, tomando por base os ensaios clínicos prospectivos já publicados. As populações estudadas podem ser diferentes, assim como seus riscos relativos e absolutos, critérios de 


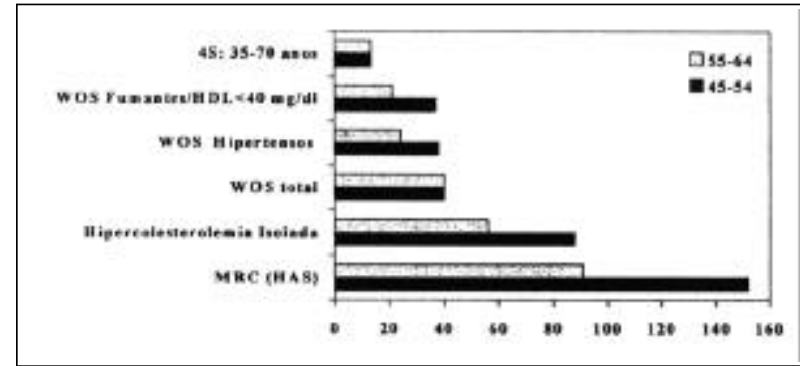

Fig. 2 - Número de pacientes necessários para tratar a fim de prevenir um evento em vários estudos prospectivos placebo-controlados. MRC-Medical Research Council; WOS- West of Scotland; HAS- hipertensão arterial sistêmica. Adaptado de Shepherd et al, XI Simpósio Internacional de Aterosclerose, Paris, França, 1997.

inclusão e exclusão e desfechos primários e secundários pré-especificados. Em relação aos grandes ensaios com vastatinas, somente os estudos CARE e LIPID, pela semelhança no desenho, permitem algumas comparações diretas. Diferentes desfechos pré-especificados e a concomitância de outras terapias de ação cardiovascular (tab. I) também dificultam as comparações diretas.

O custo aproximado do tratamento de 40 pacientes assintomáticos, hipercolesterolêmicos de meia idade, com $40 \mathrm{mg}$ de pravastatina por cinco anos para impedir uma morte coronária ou infarto não fatal é de aproximadamente $190 \mathrm{mil}$ reais ou cerca de 38 mil reais por ano. Nos EUA, o custo correspondente para prevenção de uma morte cardíaca com a pravastatina seria equivalente a aproximadamente $686 \mathrm{mil}$ dólares em cinco anos ${ }^{53}$. Os resultados recentemente divulgados do estudo AF/TEXCAPS pareceram estender a abrangência dos efeitos benéficos das vastatinas a um contingente significativamente maior de pessoas, donde sérias implicações de ordem econômica podem resultar. A rigor, a fim de maximizar sua custo-eficácia, a decisão de tratar um indivíduo assintomático com drogas hipolipemiantes deve se basear nos resultados dos ensaios clínicos prospectivos referentes a populações com características próximas às do indivíduo em questão e procurar definir previamente o risco absoluto de um evento cardíaco, em cada caso particular, num determinado período de tempo. O benefício obtido deverá ser tanto maior quanto maior o risco absoluto daquele indivíduo. Para esta última finalidade, desnecessária em pacientes previamente coronariopatas, e portanto de alto risco, dispõem-se de tabelas já elaboradas, como as do consenso europeu para tratamento de dislipidemia ${ }^{54}$ ou as tabelas de risco de Sheffield ${ }^{55}$, tomando por base os modelos de Framingham. O benefício individual absoluto obtido com o tratamento seria equivalente ao produto entre a redução no risco relativo de um desfecho primário, conforme descrito no ensaio clínico prospectivo, e a estimativa do risco absoluto pré-tratamento determinada pela tabela escolhida. Ainda que relativamente alto, o NNT assim estimado para estudos de prevenção primária, como o WOSCOPS, compara-se favoravelmente com o de estudos consagrados ${ }^{56}$, como o MRC para o tratamento de hipertensão arterial (fig. 2) tanto em idosos ${ }^{57}$ como em indivíduos mais jovens ${ }^{58}$.

É possível que métodos não invasivos como a medida da espessura íntima-média carotídea ${ }^{52}$ e a detecção de calcificações coronárias por tomografia computadorizada ultra-rápida ${ }^{59}$ venham a se converter em instrumentos custo-eficazes para refinar a indicação do uso de vastatinas mediante a detecção de graus mais sutis de agressão vascular em indivíduos mais jovens, com perspectivas de uso da medicação a longo prazo e naqueles com níveis limítrofes de lipídeos plasmáticos ou comorbidades indicativas de maior risco.

Os efeitos favoráveis das vastatinas são consistentes ao longo de todas as categorias de risco? - Diversos subgrupos de pacientes foram pré-especificados nos grandes ensaios clínicos e se justifica uma discussão mais detalhada ao longo dessas categorias, de modo a extrair o máximo de conclusões sobre as condutas mais custo-eficazes.

Pacientes diabéticos - Uma análise post-hoc do estudo $4 \mathrm{~S}$, ocupando-se apenas desta categoria de pacientes, revelou reduções no risco relativo de um desfecho primário similares às do restante do grupo, mas com benefício absoluto bem superior: a cada 100 pacientes hipercolesterolêmicos sobreviventes de um infarto tratados por seis anos com sinvastatina, nove eventos cardiovasculares maiores foram evitados em cada 29 pacientes não diabéticos, contrastando com 24 eventos evitados em cada 46 diabéticos ${ }^{60}$. Cerca de $14 \%$ da população do estudo CARE era composta de diabéticos e para estes, a redução no risco relativo foi similar à observada no restante do grupo. Nos pacientes aparentemente saudáveis, com níveis medianos de LDLc integrantes do estudo AF/TEXCAPS, a redução de $43 \%$ no risco de um desfecho primário foi bem superior à encontrada no restante dos pacientes, mas somente 143 dentre os 6605 integrantes eram diabéticos, deixando margem, portanto, a especulações sobre a insuficiência de casos para uma análise estatística segura.

Com base nos dados disponíveis é possível, com boa margem de segurança, concluir pela possibilidade concreta de se obter extensão aos diabéticos do mesmo benefício conferido pelas vastatinas aos não diabéticos.

Pacientes do sexo feminino - Os níveis de colesterol total e LDLc elevam-se nas mulheres a partir da menopausa, onde valores médios de, respectivamente, $260 \mathrm{mg} / \mathrm{dL}$ e $155 \mathrm{mg} / \mathrm{dL}$ são atingidos por volta dos 65-69 anos. A partir dos 65 anos, a prevalência de hipercolesterolemia nas mulheres é superior à dos homens e, somado este fato à maior expectativa de vida no sexo feminino, pode haver mais mulheres que homens em risco a partir desta faixa etária ${ }^{61}$. A tendência observada de queda dos níveis de LDLc em idades mais avançadas, infelizmente, não contribui para reduzir a incidência dos casos de coronariopatia, neutralizada que é pela contribuição do envelhecimento como fator de risco independente e devastador. Portanto, a terapia redutora de colesterol nas mulheres pós-menopausa seria, em tese, altamente custo-eficaz em função da possibilidade de gerar expressivas reduções de risco absoluto.

As evidências fornecidas pelos grandes ensaios clínicos para se avaliar o efeito das vastatinas sobre a redução de risco nas mulheres são ainda incompletas. Não obstante 
as tentativas de se recrutar um contingente significativo, capaz de responder às questões formuladas, as mulheres representam ainda, menos de 20 mil pacientes/ano de observação, pouco mais que a décima parte do sexo masculino, a grande maioria das quais, em estudos de prevenção secundária.

O estudo 4S demonstrou que a mulher hipercolesterolêmica sobrevivente de um IM beneficia-se das vastatinas tanto quanto os homens, ao menos em relação à prevenção da totalidade de eventos ou cirurgias de revascularização. Neste estudo, para o qual foram recrutadas 827 mulheres, não se observou redução das mortalidades total e coronária, possivelmente devido ao pequeno número de mortes registrado ${ }^{62}$. No estudo CARE, a redução no risco relativo de um desfecho primário foi bem mais expressiva que nos homens, apesar dos níveis medianos de LDLc bem inferiores ao das pacientes do $4 \mathrm{~S}$.

Os resultados do estudo LIPID não demonstraram diferenças estatisticamente significativas na incidência de eventos entre as mulheres dos grupos tratado e placebo. Resta saber se a análise de subgrupos ainda a divulgar poderá revelar benefícios para alguma categoria de mulheres, como por exemplo, as hipercolesterolêmicas.

Existem até o momento, apenas 997 mulheres avaliadas num único estudo de prevenção primária, o $\mathrm{AF} /$ TEXCAPS, onde a redução em $25 \%$ dos níveis médios de LDLc em torno de $145 \mathrm{mg} / \mathrm{dL}$ produziu uma expressiva redução de $46 \%$ no risco de um evento coronário, superior àquela observada nos homens. Estima-se neste tipo de paciente do sexo feminino, um risco de eventos coronários futuros em torno de cinco a $15 \%$ em 10 anos ${ }^{63}$. Portanto, um resultado tão positivo, só pode ser interpretado aceitando-se a possibilidade de efeitos não ligados à redução do colesterol ou admitindo-se alguma inconsistência estatística nos achados. Esta última hipótese não pode ser levada em conta sem o exame detalhado do estudo, o que deverá ocorrer em 1998 por ocasião de sua publicação.

Quaisquer discussões sobre medidas de prevenção primária ou secundária de eventos coronários em mulheres não podem prescindir de considerações sobre uma alternativa terapêutica exclusiva do sexo feminino, representada pela terapia de reposição hormonal (TRH). Presentemente, há dois estudos prospectivos placebo-controlados em andamento com o objetivo de definir o real papel preventivo da TRH, com resultados previstos para o próximo século. Até o momento, dispõe-se apenas de evidências oriundas de estudos observacionais, sujeitas a interpretações subjetivas, mas fortemente indicativas de uma redução de até $45 \%$ na incidência de eventos coronários em mulheres tratadas com TRH ${ }^{64-66}$.

Dois estudos duplo-cegos recentes compararam diretamente vastatinas e TRH isoladamente ou em combinação $0^{67,68}$. As conclusões destes dois estudos levantam a possibilidade de benefícios equivalentes sobre o perfil lipídico, desde que consideradas conjuntamente as ações sobre HDLc e LDLc. A possibilidade de combinar-se as duas terapias em doses menores possibilitaria, segundo um desses estudos, efeitos sinérgicos com amplo potencial na prevenção de eventos cardiovasculares.

Conclui-se, portanto, pela necessidade de estudar mais a fundo, nas pacientes do sexo feminino, o significado clínico da administração de vastatinas, seja como única medida farmacológica redutora do colesterol, ou comparada e até mesmo associada em doses menores, a terapias alternativas e mais econômicas, especialmente na prevenção primária. Até o presente momento, podem ser considerados altamente promissores, mas não inequívocos os benefícios derivados da administração de vastatinas em mulheres normocolesterolêmicas, tanto em prevenção primária como secundária.

Pacientes idosos - Parece hoje fora de qualquer dúvida, a necessidade de se obter redução dos níveis de LDLc na população idosa. Todos os grandes ensaios clínicos demonstram a utilidade das vastatinas e sua maior custo-eficácia nos pacientes de até 75 anos, nos quais o benefício absoluto pode superar aquele obtido em pacientes mais jovens ${ }^{2,3,61}$. Pacientes acima deste limite, entretanto, não foram estudados em número suficiente para permitir conclusões. No estudo CARE, cerca de 1116 pacientes entre 65 e 75 anos chegaram ao final da fase de acompanhamento com idades entre 70 e 80 anos. Nestes, a redução no risco de um desfecho primário foi de 32\%, comparada a uma redução de $19 \%$ entre os pacientes mais jovens (diferença não-significativa). Entretanto, como a proporção de eventos nos idosos era bem maior, a redução absoluta de 5,2\% no risco de eventos comparou-se muito favoravelmente com a redução absoluta de $2,1 \%$ nos mais jovens ${ }^{69}$. Estes dados sugerem a possibilidade de extensão do benefício absoluto aos indivíduos $>80$ anos, embora somente um ensaio clínico controlado possa oferecer a resposta.

O desafio da aderência à terapia redutora do colesterol - A perspectiva de redução no risco de acidentes cardiovasculares oferecida pelo uso de inibidores da HMGCoA redutase não se compatibiliza com uso ocasional, irregular ou intermitente. Entretanto, parece faltar até mesmo aos médicos, o grau de informação necessária para persuadir o paciente da necessidade de uso contínuo da medicação. Malgrado, por exemplo, a ampla divulgação na Escócia dos resultados do estudo WOSCOPS, um significativo percentual de indivíduos era aconselhado por seus médicos assistentes a interromper o consumo de pravastatina, por já se encontrarem normalizados os níveis de colesterol (Shepherd J, comunicação pessoal).

Em prevenção secundária, o quadro não é menos desanimador: O estudo HERS, atualmente em andamento, tem por objetivo primário estudar o efeito da terapia de reposição hormonal em mulheres pós-menopausa sobreviventes de um episódio coronário agudo. A fase de recrutamento, já concluída, possibilitou a publicação de um estudo visando avaliar que proporção de mulheres enquadrava-se nas recomendações propostas pelos ATP I e II do NCEP, respectivamente, $\mathrm{LDLc}<130 \mathrm{mg} / \mathrm{dL}$ e $\mathrm{LDL}<100 \mathrm{mg} / \mathrm{dL}^{70-72}$. Nesta amostra de 2763 mulheres, vale ressaltar, $47 \%$ das pacientes consumiam um redutor de colesterol, representa- 
do, em $36 \%$ dos casos, por um inibidor da redutase. Entretanto, 63\% delas não alcançavam sequer os objetivos propostos pelo ATP I, quanto mais os do ATP II, somente cumpridos por $9 \%$ das mulheres. Tais metas, por menos realistas que possam parecer, sugerem, na pior das hipóteses, uma atitude por demais complacente em relação à consciência do risco envolvido na manutenção de níveis elevados de LDLc após um insulto isquêmico agudo.

O estudo ASPIRE apresentou uma pesquisa sobre prevenção secundária de eventos coronários, realizada no Reino Unido, em 2.583 sobreviventes de um episódio agudo, igualmente distribuídos entre ambos os sexos ${ }^{73}$. Após a alta hospitalar, somente cerca de $10 \%$ dos pacientes tomavam algum redutor de colesterol (não está comprovada a eficácia das vastatinas nos primeiros três meses após um episódio agudo), mas após seis meses, dentre as pessoas em uso de terapia hipolipemiante, cerca de $57 \%$ dos homens e $69 \%$ das mulheres exibiam colesterol total $>220 \mathrm{mg} / \mathrm{dL}$. Em outro expressivo exemplo, mais da metade dos pacientes aos quais foram prescritos inibidores da redutase, já os haviam suspenso ao fim do $1^{\circ}$ ano de uso ${ }^{74}$.

Certamente, o alto custo individual da terapia representa fator de desestímulo à continuidade do tratamento, mas não justifica isoladamente tão elevado índice de abandono, tampouco compreensível por qualquer perfil individual desfavorável de tolerância clínica. Afinal, no berço do estudo WOSCOPS, a pravastatina era fornecida gratuitamente e mesmo assim, a taxa de abandono foi elevada.

\section{Conclusão}

Segundo a American Heart Association, a doença coronária terá representado, em 1997, um custo de 90,9 bilhões de dólares, divididos entre 47,5 bilhões em custos diretos e 43,4 bilhões em perda de produtividade. Deste montante, o gasto com medicação responderia por apenas 2,7 bilhões de dólares, o equivalente a $6 \%$ dos custos diretos e $3 \%$ dos custos totais ${ }^{75}$.
Faz-se imperativo, portanto, um esforço educacional dirigido à população médica, particularmente não cardiologista, sobre a importância da prevenção primária e secundária da doença isquêmica com inibidores da HMG-CoA redutase. São os clínicos gerais, endocrinologistas, geriatras, médicos de família e médicos de atendimento primário, os principais responsáveis pelo atendimento ao maior número de pacientes em risco. Pelo lado da indústria farmacêutica, à semelhança do ocorrido com os inibidores da enzima conversora da angiotensina, espera-se uma redução do preço final dos medicamentos, por conta do aumento da concorrência entre as diversas empresas e do vencimento dos prazos de vigência das patentes de fabricação.

A participação fundamental dos órgãos governamentais e representativos da classe médica em campanhas visando motivar os indivíduos a promover radicais modificações em seus hábitos de vida, parece ter obtido resultado em países com elevada prevalência da enfermidade coronária e pode, igualmente, entre nós, gerar bons frutos. Assistimos preocupados à escalada de hábitos, como tabagismo e consumo exagerado de sódio e gorduras saturadas entre as classes mais desfavorecidas, justamente as maiores consumidoras dos cronicamente escassos recursos previdenciários. Os números amplamente divulgados na imprensa comprovam o aumento na expectativa de vida da nossa população e, seguramente, tal não ocorre às custas do envelhecimento de nossa minguada classe média. Sem um esforço de conscientização da classe médica e a devida atenção a medidas preventivas para este que constitui o maior segmento da população brasileira, jamais haverá recursos para melhorar, mesmo em alguns poucos pontos percentuais, a saúde de nosso povo.

Em escala planetária, apesar de muitos percalços, a ciência parece tornar mais próximo o sonho de se resgatar, com menos revascularizações e, esperançosamente, a baixo custo, um expressivo contingente de pessoas para uma vida produtiva dentro dos limites - ainda mutáveis - impostos por nossa finitude.

\section{Referências}

1. Scandinavian Simvastatin Survival Group - Randomized trial of cholesterol lowering in 4444 patients with coronary heart disease: The Scandinavian Simvanstatin Survival Study (4S). Lancet 1994; 344: 1383-9.

2. Shepherd J, Cobbe Sm, Ford I et al - Prevention of coronary heart disease with pravastatin in men with hypercholesterolemia. N Engl J Med 1995; 333: 13017.

3. Sacks FM,Pfeffer MA, Moye LA et al - Cholesterol and recurrent events trial Investigators. N Engl J Med 1996; 335: 1001-9.

4. Pedersen TR, Kjekshus J, Olsson AJ, Cook TJ (abstr) - 4S results support AHA guidelines to reduce LDL-cholesterol to less than $100 \mathrm{mg} / \mathrm{dl}$ in patients with CHD. Circulation 1997; 96(suppl I) I-717.

5. Wong ND, Wilson PWF, Kannel W - Serum cholesterol as a prognostic factor after myocardial infarction: the Framingham Study. Ann Intern Med 1991; 115: $687-93$.

6. Sociedade Brasileira de Cardiologia - $2^{\circ}$ Consenso Brasileiro sobre Dislipidemias. Arq Bras Cardiol 1996; 67: 1-16
7. Brown BG, Zhao X-Q, Sacco DE, Alberts JJ - Lipid lowering and plaque regression: new insights into prevention of plaque disruption and clinical events in coronary disease. Circulation 1993; 87: 1781-91.

8. Eischtadt HW, Eskotter H, Hoffman I et al - Improvement of myocardial perfusion by short-term fluvastatin in coronary artery disease. Am J Cardiol 1995; 76 122A-5A.

9. Mostaza JM, del Valle Gornez Martinez M, Gallardo F, Salazar ML et al - Increase of myocardial perfusion during antilipemic treatment in patients with ischemic cardiopathy. Rev Esp Cardiol 1996; 49: 669-74.

10. Vita JA, Treasure CB, Young AC et al - Patients with evidence of coronary endothelial dysfunction as assessed by acetylcholine infusion demonstrate marked increase in sensitivity to constrictor effects of catecholamines. Circulation 1992; 85: 1390-9.

11. Zeiher AM, Drexler H, Saurbier B et al - Endothelium-mediated coronary blood flow modulation in humans: effect of age, atherosclerosis, hypercholesterolemia, and hypertension. Circulation 1992; 92: 652-62.

12. Egashira K, Hirooka Y, Kai H et al - Reduction in serum cholesterol with 
pravastatin improves endothelium-dependent coronary vasomotion in patients with hypercholesterolemia. Circulation 1994; 89: 2519-24.

13. Treasure CB, Klein L, Weintraub BS et al - Beneficial effects of cholesterol-lowering therapy on the coronary endothelium in patients with coronary artery disease. N Engl J Med 1995; 332: 481-7.

14. Haynes D, Weber R, Rutschmann B et al - Postischemic blood flow response in hypercholesterolemic patients. Hypertension 1993; 26: 497-502.

15. Anderson TJ, Meredith IT, Yeung AC et al - The effect of cholesterol-lowering and antioxidant therapy on endothelium-dependent coronary vasomotion. N Engl J Med 1995; 332: 488-93.

16. O'Driscoll G, Green D, Taylor RR - Simvastatin, an HMG-Coenzyme A reductase inhibitor, improves endothelial function within 1 month. Circulation 1997; 95 : 1126-31.

17. Buchwald H, Campos CT, Boen JR et al - Disease-free intervals after partial ileal bypass in patients with coronary heart disease and hypercholesterolaemia: report from the program on the surgical control of the hyperlipidemias ( $\mathrm{POSCH}) . \mathrm{J}$ Am Coll Cardiol 1995; 26: 351-7.

18. Packard CJ (abstr) - Relationship between LDL-C changes and CHD event reduction with pravastatin in the West of Scotland Coronary Prevention Study (WOSCOPS). Circulation 1997; 96 (suppl I) I-107.

19. West of Scotland Coronary Prevention Study Group - Influence of pravastatin and plasma lipids on clinical events in the West of Scotland Coronary Prevention Study (WOSCOPS). Circulation 1998; 97: 1440-5.

20. Anderson KM, Odell PM, Wilson PW, Kannel WB - Cardiovascular disease risk profiles - Am Heart J 1991; 121: 293-6.

21. Lennernäs H, Fager, G - Pharmacodynamics and pharmacokinetics of the HMGCoA reductase inhibitors: similarities and differences. Clin Phamacokinet 1997; 32: 403-25.

22. Knopp RH, Illingworth DR, Stern EA et al - Effect of pravastatin in the treatment of patients with type III hyperlipoproteinemia. Am J Ther 1996; 3: 213-9.

23. Vaughan CJ, Murphy MB, Buckley BM - Statins do more than just lower cholesterol. Lancet 1996; 348: 1079.

24. Lacoste L, Lam JYT, Hung J et al - Hyperlipidaemia and coronary disease: correction of the increased thrombogenic potential with cholesterol reduction. Circulation 1995; 92: 3172-7.

25. DiMinno G, Silver MJ, Cerbone AM et al - Increased fibrinogen binding to platelets from patients with familial hypercholesterolemia. Arteriosclerosis 1986; 6: 203-11.

26. Tsuda $\mathrm{Y}$, Satoh K, Kitadai M et al - Effects of pravastatin sodium and simvastatin on plasma fibrinogen level and blood rheology in type II hyperlipoproteinemia. Atherosclerosis 1996; 122: 225-33.

27. Munro E, Patel M, Chan P et al - Inhibition of human vascular smooth muscle cell proliferation by lovastatin: the role of isoprenoid intermediates of cholesterol synthesis. Eur J Clin Invest 1994; 24: 766-72.

28. Keidar S, Aviram M, Maor I et al - Pravastatin inhibits cellular cholesterol synthesis and increases low density lipoprotein receptor activity in macrophages: in vitro and in vivo studies. Br J Clin Pharmacol 1994; 38: 513-9.

29. Harper CM, Fyfe AI (abstr) - Hmg-Coa Reductase Inhibitors Block Multiple Events in Atherogenesis. Circulation 1997 (suppl I)I-177.

30. Grandaliano G, Biswas P, Choudhury GG, Abboud HE - Simvastatin inhibits PGDF-induced DNA synthesis in human glomerular mesangial cells. Kidney Int 1993; 44: 503-8.

31. Ross R, Raines EW, Bowen-Pope DF - The biology of platelet-derived growth factor. Cell 1986; 46: 155-69.

32. Hidaka Y, Eda T, Yonemoto M, Kamei T - Inhibition of cultured vascular smooth muscle cell migration by simvastatin (MK-733). Atherosclerosis 1992; 95: 8794.

33. Aviram M, Dankner G, Cogan U et al - Lovastatin inhibits low-density lipoprotein oxidation and alters its fluidity and uptake by macrophages: in vitro and in vivo studies. Metabolism 1992; 41: 229-35.

34. Giroux LM, Davignon J, Naruszewicz M - Simvastatin inhibits the oxidation of low-density lipoproteins by activated human monocyte-derived macrophages. Biochim Biophys Acta 1993; 1165: 335-8.

35. Salonen R, Nyysonen K, Porkkala-Sarataho E, Salonen JT - The Kuopio Atherosclerosis Prevention Study (KAPS): effect of pravastatin treatment on lipids, oxidation resistance of lipoproteins, and atherosclerotic progression. Am J Cardiol 1995; 76: 34C-39C

36. Kong SX, Crawford S, Gandhi SK, Seeger JD et al - Efficacy of 3-hydroxy-3methylglutaryl coenzyme A reductase inhibitors in the treatment of patients with hypercholesterolemia: a meta-analysis of clinical trials. Clin Therapeut 1997; 19: 778-97.

37. Nawrocki JW, Weiss SR, Davidson MH et al - Reduction of LDL-cholesterol by $25 \%$ to $60 \%$ in patients with primary hypercholesterolemia by atorvastatin, a new HMG-CoA reductase inhibitor. Arterioscler Thromb Vasc Biol 1995; 15: 67882.

38. Marais AD, Firth JC, Bateman ME et al - Atorvastatin: An effective lipid-modify- ing agent in familial hypercholesterolemia. Arterioscler Thromb Vasc Biol 1997; 17: 1527-31.

39. Schulte K-L, Beil S - Efficacy and tolerability of fluvastatin and simvastatin in hypercholesterolaemic patients: a double-blind, randomized, parallel-group comparison. Clin Drug Invest 1996; 12: 119-26.

40. Haasis R, Berger J, Anderson F et al - A pharmacoeconomic evaluation of fluvastatin and lovastatin in primary hypercholesterolemia. Br J Med Econ 1996; 10: 145-57.

41. Holme I - An analysis of randomized trials evaluating the effect of cholesterol reduction on total mortality and coronary heart disease incidence. Circulation 1990; 82: 1916-24

42. Goldbourt U, Holtzman E, Neufeld HN - Total and high density lipoprotein cholesterol in the serum and risk of mortality: evidence of a threshold effect. Br Med J 1985; 290: 1239-43

43. Sacks FM, Gibson M, Rosner B et al - The influence of pretreatment low density lipoprotein cholestrol concentrations on the effect of hypocholesterolemic therapy on coronary atherosclerosis in angiographic trials. Am J Cardiol 1995; 76: $78 \mathrm{C}-85 \mathrm{C}$.

44. Sacks FM, Pfeffer MA, Moye LA et al-Cholesterol and recurrent events trial Investigators. N Engl J Med 1996; 335: 1001-9.

45. Marchioli R, Marfisi RM, Carinci F, Tognomi G - Meta-analysis, clinical trials, and transferability of research results into practice. Arch Intern Med 1996; 156: 1158-72.

46. The Post Coronary Artery Bypass Graft Trial Investigators - The effect of aggressive lowering of low-density lipoprotein cholesterol levels and low-dose anticoagulation on obstructive changes in saphenous-vein coronary-artery bypass grafts. N Engl J Med 1997; 336: 153-62.

47. Pedersen TR, Olsson AG, Fergeman O et al - Lipoporotein changes and reduction in the incidence of coronary heart disease events in the Scandinavian Simvastatin Survival Study (4S). Circulation 1998; 97: 1453-61.

48. Sacks FM, Moyé LA. Davis BR et al - Relationship between plasma LDL concentrations during treatment with pravastatin and recurrent coronary events in the cholesterol and recurrent events trial. Circulation 1998; 97: 1446-52.

49. Ouchi $\mathrm{Y}$ et al (abstr) - Serum cholesterol lowering by pravastatin reduces cardiovascular events in the elderly with hypercholesterolemia: Overall and age-related analyses of the results from the PATE Study. Circulation 1997; 96(suppl I) I-66.

50. Jönsson B, Johannesson M, Kjeshush J et al - Cost-effectiveness of cholesterol lowering. Results from the Scandinavian Simvastatin Survival Study (4S). Eur Heart J 1996; 17: 1001-7.

51. Samani NJ, de Bono DP (letter) - Prevention of coronary heart disease with pravastatin. N Engl J Med 1997; 336: 962.

52. Simon A, Megnien JL, Levenson J - Coronary risk and treatment of hypercholesterolemia. Circulation 1997; 96: 2449-52.

53. Rogers S (letter) - Prevention of coronary heart disease with pravastatin. N Eng J Med 1997; 336: 962

54. Pyöralla K, De Backer G, Graham I, Poole-Wilson P, Wood D, on behalf of the Task Force - Prevention of coronary heart disease in clinical practice: recommendations of the Task Force of the European Society of Cardiology, European Atherosclerosis Society and European Society of Hypertension. Eur Heart J 1994; 15: $1300-31$

55. Haq IU, Jackson PR, Yeo WW, Ramsay LE-Sheffield Risk and treatment table for cholesterol lowering for primary prevention of coronary heart disease. Lancet 1995; 366: 1467-71

56. West of Scotland Coronary Prevention Study - identification of high-risk groups and comparison with other cardiovascular intervention trials. Lancet 1996 Nov 16; 348: 1339-42.

57. MRC Working Party - Medical Research Council trial of treatment of hypertension in older adults: principal results. Br Med J 1992; 15: 304: 405-12.

58. Medical Research Council Working Party - MRC trial of treatment of mild hypertension: principal results. Br Med J 1985; 291: 97-104.

59. Rumberger JA, Sheedy PF, Breen JF et al - Electron beam computed tomography and coronary artery disease: scanning for coronary artery calcification. Mayo Clin Proceedings 1996; 71: 369-77.

60. Pyoräla K, Pedersen TR, Kjekshus J et al - Cholesterol lowering with simvastatin improves prognosis of diabetic patients with coronary heart disease. Diabetes Care 1997; 20: 614-20.

61. Kannel WB - Range of serum cholesterol values in the population developing coronary artery disease - Am J Cardiol 1995; 76: 69C-77C

62. Miettinen TA, Pyörälä K, Olsson AG et al - Cholesterol-lowering therapy in women and elderly patients with myocardial infarction or angina pectoris. Findings from the Scandinavian Simvastatin Survival Study (4S). Circulation 1997; 96: 4211-8

63. Pyöralla K, De Backer G, Graham I, Poole-Wilson P, Wood D, on behalf of the Task Force - Prevention of coronary heart disease in clinical practice: recommendations of the Task Force of the European Society of Cardiology, European Ath- 
erosclerosis Society and European Society of Hypertension. Eur Heart J 1994; 15: $1300-31$.

64. Grady D, Rubin S, Petitti DB et al - Hormone therapy to prevent disease and prolong life in postmenopausal women. Ann Intern Med 1992; 117: 1016-37.

65. Stampfer MJ, Colditz GA, Willet WC - Menopause and heart disease: a review. Ann NY Acad Sci 1990; 592: 193-206.

66. Lobo RA - Clinical review 27: effects of hormonal replacement on lipids and lipoproteins in postmenopausal women.. J Clin Endocrinol Metab 1991; 73: 92530 .

67. Darling GM, Jones JA, McCloud PI, Davis SR - Estrogen and progestin compared with simvastatin for hypercholesterolemia in postmenopausal women. N Engl J Med 1997; 337: 595-601.

68. Davidson MH, Testolin LM, Maki KC et al - A comparison of estrogen replacement, pravastatin, and combined treatment for the management of hypercholestrolemia in postmenopausal women. Arch Intern Med 1997; 157: 1186-92.

69. Sacks FM, Pfeffer MA, Braunwald E (letter) - N Engl J Med 1997; 336: 962.
70. Schrott $\mathrm{HG}$ et al - Adherence to national cholesterol education program treatment goals in postmenopausal women with heart disease: The Heart and Estrogen/Progestin Replacement Study (HERS). JAMA 1997; 277: 1281-6.

71. Report of the National Cholesterol Education Program Expert Panel on Detection, Evaluation, and Treatment of High Blood Cholesterol in Adults. The Expert Panel. Arch Intern Med 1988; 148: 36-69.

72. Sempos CT, Cleeman JI, Carroll MD et al - Prevalence of high blood cholesterol among US adults. An update based on guidelines from the second report of the National Cholesterol Education Program Adult Treatment Panel. JAMA 1993 269: 3009-14.

73. ASPIRE Steering Group - A British Cardiac Society survey of the potential for the secondary prevention of coronary disease: ASPIRE (Action on Secondary Prevention through Interventions to Reduce Events). Heart 1996; 75: 334-42.

74. Simons LA, Levis G, Simon J - Apparent discontinuation rates in patients prescribed lipid-lowering drugs. Med J Aust 1996; 164: 208-11.

75. Gotto AM - Cholesterol management in theory and practice. Circulation 1997 96: 4424: 30 . 